\title{
Structure Design and Movement Simulation Research of Bionic Artificial Leg
}

\author{
Hualong Xie ${ }^{1, a}$, Zheng Jia ${ }^{1, b}$, Deming Zhao ${ }^{1, c}$ \\ ${ }^{1}$ School of Mechanical Engineering and Automation, Northeastern University, \\ Shenyang, 110819, China \\ aemail: hlxie@mail.neu.edu.cn, bemail: jiazhenghi@126.com, email: neudeming@163.com
}

Keywords: Bionic Artificial Leg; Four-bar Bionic Knee; Genetic Algorithm;

\begin{abstract}
Bionic artificial leg is used to simulate the disabled health leg and provided ideal following gait for intelligent prosthesis test, so it raises a high demand about humanoid character and intelligent control. Referring to the parameters of human leg and the movement mechanism, the bionic artificial leg was designed and analyzed in this paper. In order to improve the humanoid character and stability of bionic artificial leg, the knee joint was designed as a four-bar linkage which was driven by two pneumatic artificial muscles and the lengths of bars were optimized by the genetic algorithm. Then, this paper analyzed the bionic artificial leg movement and established the system model. Finally, the stability and humanoid character of system is verified by the MATLAB movement simulation.
\end{abstract}

\section{Introduction}

This year, with German chancellor Angela Merkel visiting China, the topics about industry 4.0 or robots are turning into even hotter. In essence, the transition from the era of industry 3.0 to 4.0 is a transformation and penetration process from the existing technology to intelligence and network. The conception of BRHL, for example, is combining with the mature technology of humanoid robot to design an ideal experimental platform which will bring convenience to the research and development of intelligent prosthetic limb and save costs [1]. So that it is significant to guarantee the humanoid performance and stability of the bionic artificial leg for the research of BRHL [2].

In this paper, the bionic artificial leg was designed based on the parameters of the human leg and the movement mechanism. CW Radcliffe [3], Zhang PY et al [4] have done a lot of research about the multi axis knee joint and confirmed that the J-curve could be used to evaluate the design of knee. In order to improve the humanoid performance and stability of the design, the knee joint was designed as a four-bar linkage which was driven by two pneumatic artificial muscles which have been widely applied in biped robots design now [5][6]. The length of bars were optimized by the genetic algorithm (GA) [7][8]. Then, this paper analyzed the artificial leg movement and established the system model. Finally, the stability and humanoid character of system is verified by the Simulink simulation, the design can satisfy the human knee joint ICR path J-curve commendably.

\section{Structure design of bionic artificial leg}

The normal physiological structure of human leg is mainly composed of hip, thigh, leg and foot four parts, as shown in Figure 1. The hip belongs to enarthrosis and can realize rotation, flexion and extension movement. The flexion and extension movements of knee and ankle are driven by muscle groups through the joint point, which are controlled by the brain nervous system. Under the premise of satisfying the reasonable intelligent prosthetic following trajectory, in order to facilitate the control of bionic artificial leg movement, the structure design was simplified slightly in this paper. Hip and ankle joints, for example, are driven by mature servo motor and the metatarsal is replaced by prosthetic foot. 


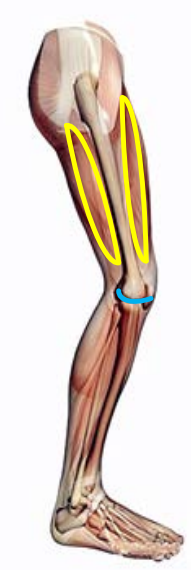

Fig.1. Bionic artificial leg physical prototype

The knee joint is more complex, mainly composed of femur, tibia, meniscus, cruciate ligament, muscle groups and nervous system, as shown in Figure 2. It is obvious that the contact surfaces of femur, tibia and meniscus are irregularly shaped, rolling and sliding movement coexist in that and the moving trajectory of instant center of rotation (ICR) is changed as the J-curve, which will adjust the torque of between the ground reaction force and the knee joint and influence the force between the hip and knee joint. As a result, it will effect the stability and energy consumption of bionic artificial leg directly during walking. Learning from relevant literature, this paper designed a four-bar bionic knee joint and employed pneumatic artificial muscles which have caught widespread attention from domestic and international scholars as joint driver.
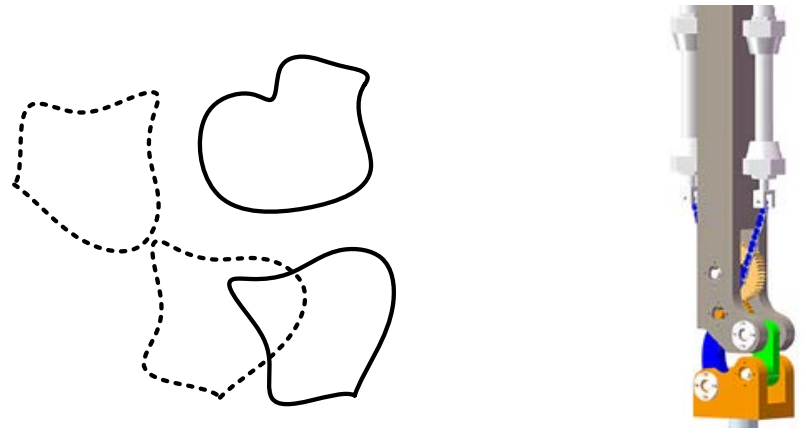

Fig.2. Knee joint structure diagram

\section{Movement modelling of bionic artificial leg}

The kinematics analysis of the bionic artificial leg emphatically studies the movement relation among joint coordinate systems and the mapping relation between the end position or the mass centers of parts and the base coordinate system, as shown in figure 3. Because of the relatively complex knee joint structure, this paper analyzed it separately to solve the movement relationship between human knee joint angle and the drive sprocket angle and to solve the coordinates of ICR.

Transforms matrixes of each joint coordinate system are shown as follows:

$$
\begin{aligned}
{ }^{0} T_{1} & =\left[\begin{array}{cccc}
\cos \theta_{1} & -\sin \theta_{1} & 0 & 0 \\
\sin \theta_{1} & \cos \theta_{1} & 0 & 0 \\
0 & 0 & 1 & 0 \\
0 & 0 & 0 & 1
\end{array}\right] \\
{ }^{0} T_{3} & =\left[\begin{array}{cccc}
\sin \left(\theta_{1}+\theta_{2}\right) & \cos \left(\theta_{1}+\theta_{2}\right) & 0 & l_{9} \cos \left(\theta_{1}-\vartheta_{1}\right)+l_{1} \sin \theta_{1} \\
-\cos \left(\theta_{1}+\theta_{2}\right) & \sin \left(\theta_{1}+\theta_{2}\right) & 0 & l_{9} \sin \left(\theta_{1}-\vartheta_{1}\right)-l_{1} \cos \theta_{1} \\
0 & 0 & 1 & 0 \\
0 & 0 & 0 & 1
\end{array}\right]
\end{aligned}
$$




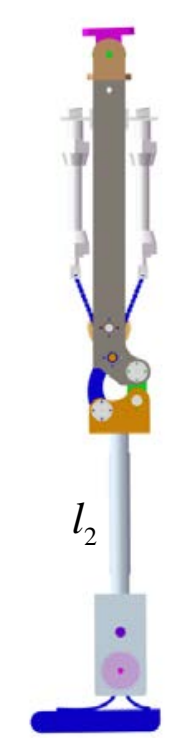

Fig.3. Bionic artificial leg motion analysis diagram

The mass centers of each part are shown as follows:

$$
\begin{aligned}
& G_{1}={ }^{0} T_{1} G_{10}=\left[\begin{array}{cccc}
\cos \theta_{1} & -\sin \theta_{1} & 0 & 0 \\
\sin \theta_{1} & \cos \theta_{1} & 0 & 0 \\
0 & 0 & 1 & 0 \\
0 & 0 & 0 & 1
\end{array}\right]\left[\begin{array}{c}
0 \\
-\frac{l_{1}}{2} \\
0 \\
1
\end{array}\right]=\left[\begin{array}{c}
\frac{l_{1}}{2} \sin \theta_{1} \\
-\frac{l_{1}}{2} \cos \theta_{1} \\
0 \\
1
\end{array}\right] \\
& G_{2}={ }^{0} T_{3} G_{20}=\left[\begin{array}{c}
\frac{l_{2}}{2} \sin \left(\theta_{1}+\theta_{2}\right)+l_{\vartheta} \cos \left(\theta_{1}-\vartheta_{1}\right)+l_{1} \sin \theta_{1} \\
-\frac{l_{2}}{2} \cos \left(\theta_{1}+\theta_{2}\right)+l_{\vartheta} \sin \left(\theta_{1}-\vartheta_{1}\right)-l_{1} \cos \theta_{1} \\
0 \\
1
\end{array}\right] \\
& G_{3}={ }^{0} T_{3} G_{30}=\left[\begin{array}{c}
\left(l_{2}+\frac{D}{2}\right) \sin \left(\theta_{1}+\theta_{2}\right)+l_{9} \cos \left(\theta_{1}-\vartheta_{1}\right)+l_{1} \sin \theta_{1} \\
-\left(l_{2}+\frac{D}{2}\right) \cos \left(\theta_{1}+\theta_{2}\right)+l_{\vartheta} \sin \left(\theta_{1}-\vartheta_{1}\right)-l_{1} \cos \theta_{1} \\
0 \\
1
\end{array}\right]
\end{aligned}
$$

In the type: $\theta_{1}$ is hip angle; $\theta_{2}$ is knee angle; $l_{1}, l_{2}$ are lengths of thigh \& shin respectively; $D$ is the load equivalent diameter; $G_{i 0}$ are the mass centers of each part in the current coordinate system.

Bionic artificial leg gait planning is completed as the normal human gaits, however, the actual human knee joint angle that represents the angle between the thigh and leg is different from driving sprocket angle. So this section will analyze the knee four-bar movement mechanism to solve the corresponding relations between them and confirm the ICR coordinate of knee joint.

Rectangular coordinate system was established as shown in in figure 4(b). According to the closed chain vector equation of four-bar, the following constraint should be satisfied:

$$
l_{21} e^{i \theta_{21}}+l_{22} e^{i \theta_{22}}-l_{23} e^{i \theta_{23}}-l_{24}=0
$$

In the type: $\theta_{21}=\alpha-\lambda \theta, \theta$ is the sprocket angle; $\theta_{2 i}$ are the angles of each bar; $\theta_{22}=\theta_{2}+\beta$, $\alpha, \beta$ are the angle transform constants. 


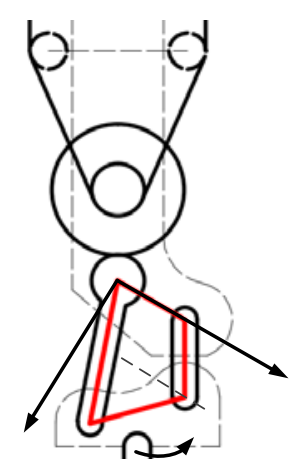

Fig.4. Knee joint motion analysis diagram

The formula above can be solved as follows:

$$
\begin{aligned}
& \theta_{2}=2 \arctan \left(\frac{a \pm \sqrt{a^{2}+b^{2}-c^{2}}}{b-c}\right)-\beta \\
& \text { In the type: } \quad \begin{aligned}
a & =-2 l_{21} l_{22} \sin (\alpha-\lambda \theta) \\
b & =2 l_{22}\left(l_{21} \cos (\alpha-\lambda \theta)-l_{24}\right) \\
c & =l_{21}{ }^{2}+l_{22}{ }^{2}-l_{23}{ }^{2}+l_{24}{ }^{2}-2 l_{21} l_{24} \sin (\alpha-\lambda \theta)
\end{aligned}
\end{aligned}
$$

According to the installation location situation of four-bar knee joint and the continuity of mechanism movement, “+” sign can be determined in the formula (6). On the basis of the theorem of three centers, the instantaneous center trajectory of knee joint can be solved easily.

\section{Movement simulation analysis}

The objective function of genetic algorithm can be written as follows:

$$
F_{(X)}=\sum_{i=1}^{m}\left[\left(x_{i c r 0}^{i}-x_{i c r}^{i}\right)^{2}+\left(y_{i c r 0}^{i}-y_{i c r}^{i}\right)^{2}\right]
$$

In the type: $\left(x_{i c r 0}, y_{i c r 0}\right)$ is the actual ICR of human knee joint; $\left(x_{i c r}, y_{i c r}\right)$ is the ICR of four-bar knee model; $m$ is the sampling number. Finally the optimized lengths of each bar which are show in table 1 are applied into artificial leg model.

Table.1. Optimized lengths of each bar

\begin{tabular}{cccc}
\hline$l_{21}$ & $l_{22}$ & $l_{23}$ & $l_{24}$ \\
\hline $82.5 \mathrm{~mm}$ & $56 \mathrm{~mm}$ & $44.5 \mathrm{~mm}$ & $44 \mathrm{~mm}$ \\
\hline
\end{tabular}

The corresponding relation of each joint angle is shown in figure 5, which is obtained by system model simulation through MATLAB. According to the mass center of each part, movement diagram was completed as shown in figure 6. The four-bar link bionic knee joint designed by our research group can satisfy the human knee joint instantaneous center path J-curve commendably, in other words, the design of bionic artificial leg had higher reasonable stability and humanoid character.
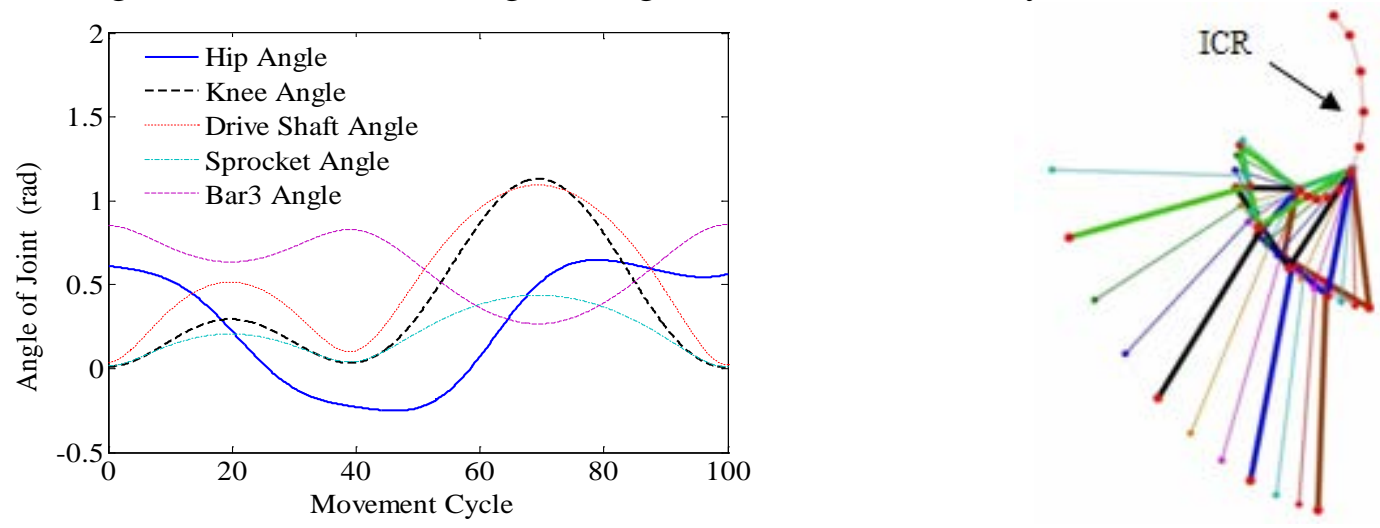

Fig.5. Movement trajectory of each part Fig.6. Movement diagram of bionic artificial leg 


\section{Conclusion}

In this paper, the bionic artificial leg was designed based on the parameters of the human leg and the movement mechanism. According to the evaluation theory of prosthetic knee joint which was built by Zhang PY, Jin DW, the movement modelling and simulation analysis were completed. The stability and flexibility of the design had been verified after the lengths which were obtained by GA were brought in the simulation model.

\section{Acknowledgement}

This paper was supported by the National Natural Science Foundation of China (Grant No.51105070, 51505072), Basic Research Project of Key Laboratory of Education Department of Liaoning Province (Grant No. LZ2015037) and the Fundamental Research Funds for the Central Universities of the Ministry of Education of China (Grant No. N140305001). We are sincerely grateful for the support.

\section{References}

[1] Xie HL, Liu ZB, Yang JY and so on. Modelling of Magnetorheological Damper for Intelligent Bionic Leg and Simulation of Knee Joint Movement Control. INT J SIMUL MODEL [J]. 2016,15(1):144-156.

[2] HL Xie, ZZ Liang, F Li, LX Guo. The Knee Joint Design and Control of Above-knee Intelligent Bionic Leg Based on Magneto-rheological Damper. International Journal of Automation \& Computing [J]. 2010, 07(3):277-282.

[3] CW Radcliffe , ME Deg. Biomechanics of knee stability control with four-bar prosthetic knees. ISPO Australia Annual Meeting[C]. Melbourne, 2003,11.

[4] Zhang PY, Jin DW and so on. Study on stability and kinematic function of above knee prostheses, Journal of Tsinghua University (Sci \& Tech) [J]. 1998(8):1-4.

[5] Wang BR, Xu H and so on. Study on four-bar linkage bionic knee joint of bipedal robots, Journal of mechanical design. 2006, 23(7):13-15.

[6] Yu HT, Guo W and so on. Design and Control on Antagonistic Bionic Joint Driven by Pneumatic Muscles Actuators. Journal of Mechanical Engineering [J]. 2012, 48(17):1-9.

[7] H Xie, S Wang, F Li. Knee Joint Optimization Design of Intelligent Bionic Leg Based on Genetic Algorithm. International Journal Bioautomation [J]. 2014,18(3).

[8] WY Lin, A GA-DE hybrid evolutionary algorithm for path synthesis of four-bar linkage, Mechanism \& Machine Theory [J]. 2010,45(8):1096-1107. 